\title{
NOTES ON SUFFICIENT CONDITIONS FOR A GRAPH TO BE HAMILTONIAN
}

\author{
MICHAEL JOSEPH PAUL \\ School of Computer Science \\ Florida International University \\ Miami, Florida 33199

\section{CARMEN BAYTAN SHERSHIN} \\ Mathematics Department \\ Ransom-Everglades School \\ Coconut Grove, Florida 33133 \\ and \\ ANTHONY CONNORS SHERSHIN \\ Mathematics Department \\ Florida International University \\ Miami, Florida 33199
}

(Received October 1, 1990 and in revised form December 8, 1990)

\begin{abstract}
The first part of this paper deals with an extension of Dirac's Theorem to directed graphs. It is related to a result often referred to as the Ghouila-Houri Theorem. Here we show that the requirement of being strongly connected in the hypothesis of the Ghouila-Houri Theorem is redundant.

The second part of the paper shows that a condition on the number of edges for a graph to be hamiltonian implies Ore's condition on the degrees of the vertices.
\end{abstract}

KEY WORDS AND PHRASEs. hamilton cycle, directed graphs, Ghouila-Houri Theorem, strongly connected digraphs, Dirac's Theorem, Ore's Theorem, edge condition.

1980 AYS SUBJECT CLASSIFICATION CODES. 05C45, $05 C 20$.

\section{EXTENDING DIRAC'S THEOREM TO DIRECTED GRAPHS}

Establishing whether or not a graph is hamiltonian is nontrivial. Unfortunately, no elegant (convenient) characterization of hamiltonian graphs exists, although several necessary or sufficient conditions are known [1]. Sufficient conditions for a graph, or digraph, to have a hamilton cycle usually take the form of implicitly requiring many edges. One such digraph result was conjectured by $C$. Berge and was proved by $M$. Alain Ghouila-Houri [2]. We shall refer to this as the G-H theorem. The hypothesis of this theorem, in addition to requiring many arcs, also stipulates that the digraph be strongly connected. One common variation of the G-H theorem extends Dirac's well known sufficient condition, for hamilton graphs, to directed graphs with the significant additional condition that such digraphs be strongly connected. In the literature this variation has sometimes been called Ghouila-Houri's Theorem [3] even though it is only an immediate corollary of the result stated in [2].

The terms used in this paper are consistent with those used by Chartrand [1]. Only simple graphs and digraphs are considered (i.e. those with no loops, parallel edges or parallel arcs).

In digraph $D$ let id(v) be the indegree of vertex $v$, od( $v)$ be the outdegree of $v$, and $p$ be the order (i.e., the number of vertices in $D$ ). 
1.1 G-H Theorem. If $D$ is a strongly connected simple digraph and if id(v) $\geq \mathrm{p} / 2$ and $\operatorname{od}(v) \geq p / 2$ for all vertices $v$ in $D$, then $D$ has a hamilton cycle. [2]

To eliminate the redundancy in the hypothesis of the G-H Theorem, we prove that only weak connectivity need be established for the G-H theorem to hold (as opposed to strong connectivity).

1.2. Lomma. If $D$ is a simple weakly connected digraph, and if for each vertex $v$, id $(v) \geq p / 2$ and od $(v) \geq p / 2$, then $D$ is strongly connected.

Proof. We shall establish the contrapositive. Suppose $D$ is not strongly connected. Then there are vertices $u$ and $v$ such that there is no dipath from $u$ to $v$. Partition the vertices of $D$ into two sets $A$ and $B$ where $A$ contains $v$ and all vertices which have a dipath to $v$, and $B$ contains all the vertices not in $A$. Since $u$ is in $B$, both sets are nonempty.

Denote the cardinality of $A$ and $B$ by $|A|$ and $|B|$, respectively. Suppose $|A| \leq|B|$. Thus $|A| \leq p / 2$ and $i d(v)$ is at most $(p / 2)-1$. Now suppose $|A|>|B|$. This implies that $|B| \leq(p / 2)-1$. The vertex u cannot be adjacent to any vertex in $A$ for, if so, it would be in A. (Moreover, $u$ cannot be adjacent to itself since $D$ is simple.) Therefore, $u$ can only be adjacent to the other vertices in $B$; that is, od $(u)<(P / 2)-1$.

Thus we have proved that if $D$ is not strongly connected, there is some vertex $w$ such that either id $(w)<\mathrm{p} / 2$ or od $(w)<\mathrm{p} / 2$.

Consequently, Dirac's theorem does generalize in an easy, immediate fashion to digraphs. This result also follows from a stronger result due to zhang Cun-Quan [4]. Moreover, since verifying that a digraph is strongly connected is nontrivial, this relaxation of the G-H Theorem becomes a useful tool to determine easily whether a digraph is hamiltonian or not.

The 1.2 Lemma is also significant in its own right because verifying that a digraph is strongly connected is an important matter. Indeed, this result, when the condition is satisfied, is easier to use when determining if a large graph is strongly connected than two other commonly used techniques: (a) computing the reachability matrix from the adjacency matrix and seeing whether or not it consists entirely of one's [5]; (b) using the depth-first search algorithm due to R.E. Tarjan [6].

We now state the refinement of (1.1) which follows immediately from it and (1.2).

1.3 Theorem. If $D$ is a simple connected digraph and if for each vertex $v, i d(v)$ $\geq p / 2$ and od $(v) \geq p / 2$, then $D$ has a hamilton cycle.

Lastly, the original Ghouila-Houri Theorem in [5] states that if $D$ is strongly connected and if $i d(v)+o d(v) \geq p$ for every vertex $v$, then $D$ has a hamilton cycle. However, this condition that the minimum total degree be at least $p$ does not guarantee that $D$ is strongly connected: Consider a digraph consisting of two complete symmetric digraphs $D$ and $K$, with $|D|$ and $|K|$ at least 2, such that $D$ and $K$ are joined completely by all possible arcs from the vertices of $D$ to the vertices of $K$. This example is mentioned in [7].

\section{A pauiliar REsult revisited.}

Several sufficient conditions for a graph to be hamiltonian have been established. The well known Dirac's condition and Ore's condition deal with the degree of the vertices of a graph. Another condition, less quoted, guarantees a hamilton cycle when there are "enough" edges [8]. Let $G$ be a simple graph having $p$ vertices and $m$ edges. Each of the following is sufficient for a graph to be hamiltonian:

Dirac's condition: For each vertex $v$ of $G$, deg $v \geq p / 2$.

ore' Condition: For each pair of nonadjacent vertices $u$ and $v$ of $G$, deg $u+$ deg $\mathrm{v} \geq \mathrm{p}$.

Edge condition: The number of edges $m \geq(1 / 2)(p-1)(p-2)+2$.

The purpose of this section is to show that the edge condition implies ore's condition, to give simple examples to show that no implication exists between Dirac's condition and the edge condition, and to show that ore's condition does not necessarily imply either Dirac's or the edge conditions:

The set diagram below (Figure 1) summarizes the relationships among hamilton graphs satisfying Ore's (O), Dirac's (D), and/or the edge condition (E). 


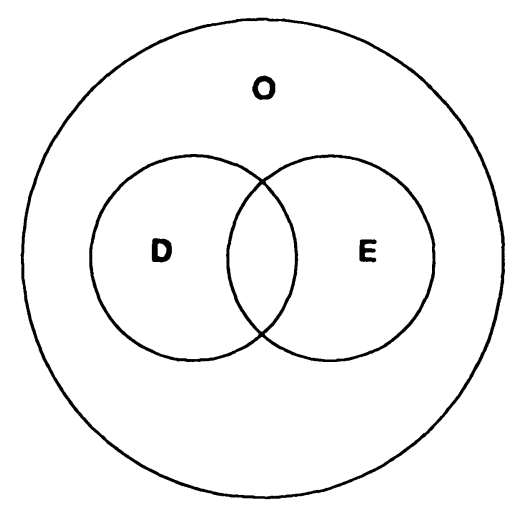

Figure 1 Venn diagram summarizing the relationships among hamilton graphs satisfying Ore's,

Dirac's, and Edge conditions.

2.1 Theorom. The edge condition implies ore's condition.

Proof. Let $G$ be a simple graph satisfying the edge condition and let $u$ and $v$ be nonadjacent vertices. The maximum number of possible edges is $(1 / 2)(p-2)(p-3)+d e g u$ $+\operatorname{deg} v$. Therefore we get $(1 / 2)(p-2)(p-3)+\operatorname{deg} u+\operatorname{deg} v \geq m \geq(1 / 2)(p-1)(p-2)+2$. This yields $\operatorname{deg} u+\operatorname{deg} v \geq(1 / 2)[(p-1)(p-2)-(p-2)(p-3)]+2=p$.

Every graph theory student learns that Dirac's condition implies ore's condition but that the converse is not true. Now we will give simple examples to show that no implication exists between Dirac's condition and the edge condition: A cycle of length four satisfies Dirac's condition, but not the edge condition; the graph of order 5 , consisting of $K_{1}$ with the addition of a vertex of degree 2 joining it to any two vertices of $K_{4}$, satisfies the edge condition but not Dirac's; a complete graph of order $\geq 3$ satisfies both Dirac's and the edge condition. Finally, Figure 2 gives an example of a graph that satisfies ore's condition (O) but does not satisfy Dirac's (D) nor the edge conditions (E).

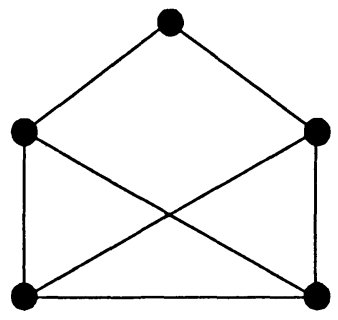

Figure 2 A graph in the set $O-(D \cup E)$

ACRONLEDGEENT. The authors thank the referee for suggesting the graph pictured in Figure 2 .

\section{REFERENCES}

[1] CHARTRAND, G., Introductory Graph Theory, Dover, 1985.

[2] GHOUILA-HOURI, M.A., Une condition suffisante d'existence d'un circuit hamiltonian, C.R. Acad. Sci. Paris 251, 1960, 495-497.

[3] WILSON, R., Introduction to Graph Theory, Longman, 1972, 107.

[4] CUN-QUAN, z. Discrete Math. 41, 1982, 79-96.

[5] HARARY, F., NORMAN, R., and CARTWRIGHT, D., structural Models: An introduction to the Theory of Directed Graphs, Wiley, 1965, 137.

[6] AHO, A., HOPCROFT, J., and ULLMAN, J.' The design and Analysis of Computer Algorithms, Addison-Wesley, 1974, 189-195.

[7] BERMOND, J.C., and THOMASSEN, C., CYCles in Digraphs - A Survey, Journal of Graph Theory 5, 1981, 1-43.

[8] BERGE, C., Graphs and Hypergraphs, North-Holland, 1973, 212-213. 


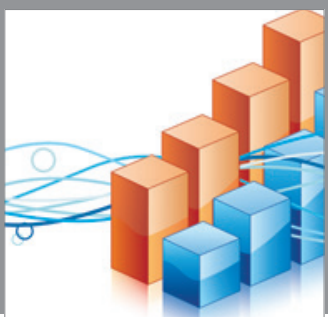

Advances in

Operations Research

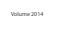

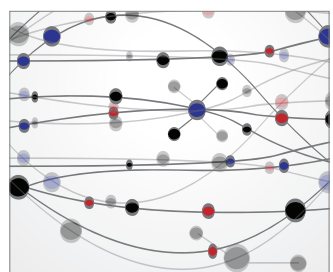

\section{The Scientific} World Journal
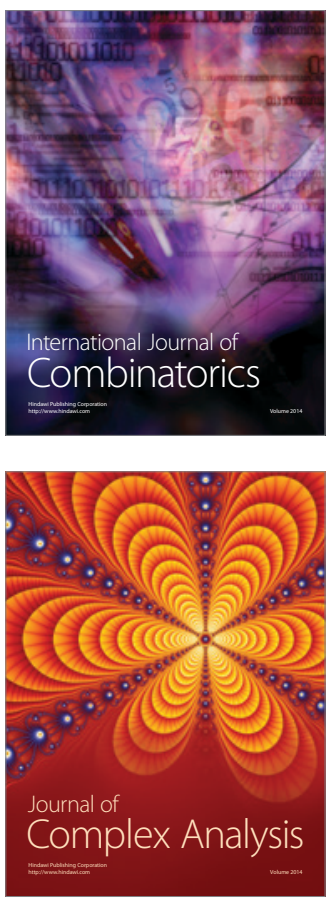

International Journal of

Mathematics and

Mathematical

Sciences
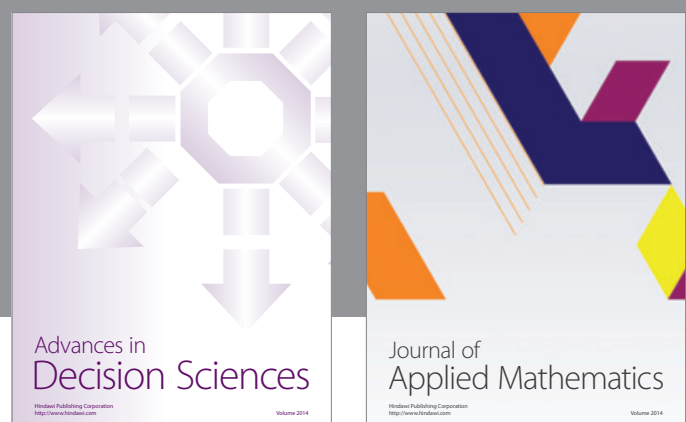

Journal of

Applied Mathematics
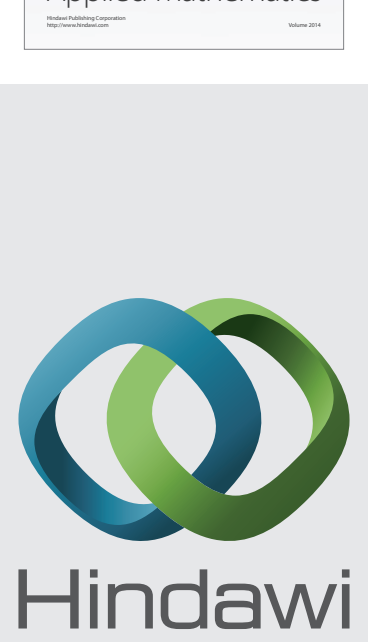

Submit your manuscripts at http://www.hindawi.com
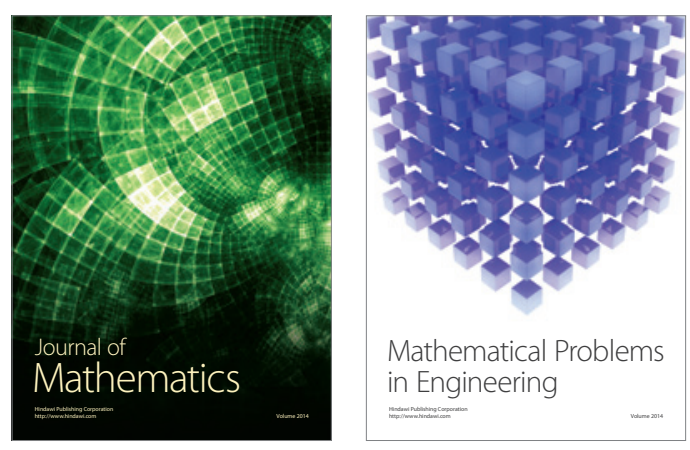

Mathematical Problems in Engineering
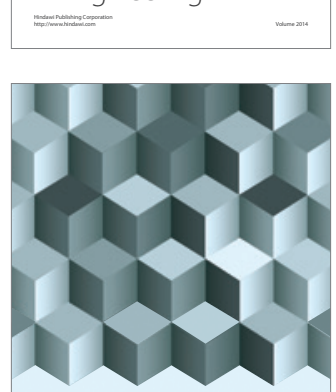

Journal of

Function Spaces
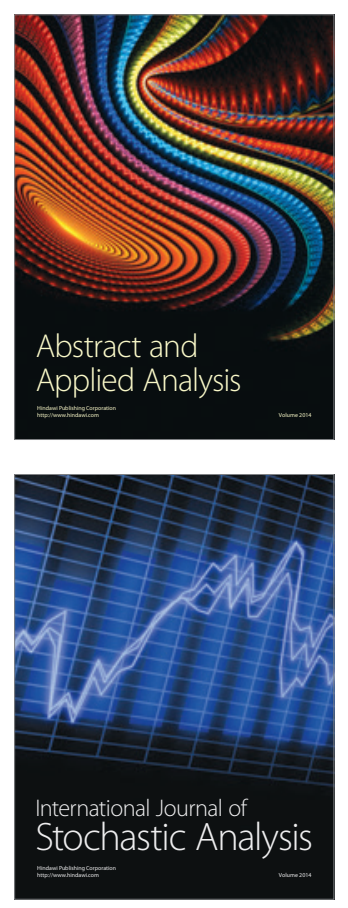

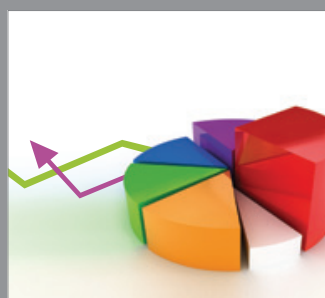

ournal of

Probability and Statistics

Promensencen
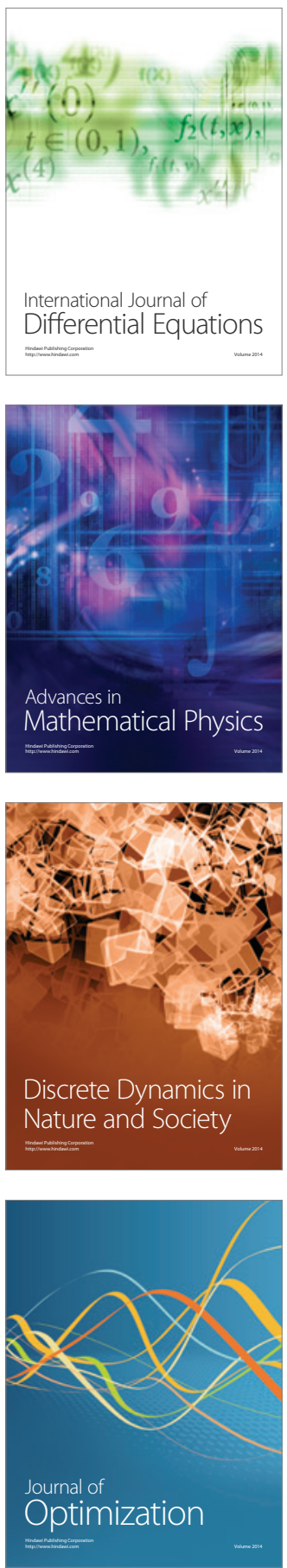